\title{
Solutions of Some Difference Equations Systems and Periodicity
}

\author{
E. M. Elsayed ${ }^{1,2}$, Faris Alzahrani ${ }^{1}{\text { Ibrahim } \text { Abbas }^{1} \text { and N. H. Alotaibi }}^{1}$ \\ ${ }^{1}$ Mathematics Department, Faculty of Science, \\ King Abdulaziz University, P. O. Box 80203, \\ Jeddah 21589, Saudi Arabia. \\ ${ }^{2}$ Department of Mathematics, Faculty of Science, \\ Mansoura University, Mansoura 35516, Egypt. \\ E-mails: emmelsayed@yahoo.com, faris.kau@hotmail.com, \\ n.h.ob@hotmail.com.
}

\begin{abstract}
In this article, analysis and investigation have been conducted on the periodic nature as well as the type of the solutions of the subsequent schemes of rational difference equations$$
x_{n+1}=\frac{1 \pm z_{n}}{y_{n-1}}, y_{n+1}=\frac{1 \pm x_{n}}{z_{n-1}}, z_{n+1}=\frac{1 \pm y_{n}}{x_{n-1}},
$$

with a nonzero real numbers initial conditions.

Keywords: difference equation, periodic solutions, system of difference equations. Mathematics Subject Classification: 39A10.
\end{abstract}

\section{Introduction}

Difference equations have normally been shown as discrete analogues as well as numerical solutions of differential and delay differential equations having some important uses in scientific areas such as, ecology, physics, economy, biology, etc. Currently, expanding concern has obviously been conducted on the study of qualitative analysis of rational difference equations and systems of difference equations. Even though, in form, difference equations look like to be elementary, it is quit complicated to be analyzed and understood thoroughly the nature of their solutions. see [1]-[23] and the references cited therein. 
A great number of researchers have examined periodic solutions of a difference equations , and different approaches have been provided for the existence and qualitative properties of the solutions.

$$
x_{n+1}=\frac{m}{y_{n}}, \quad y_{n+1}=\frac{p y_{n}}{x_{n-1} y_{n-1}},
$$

was studied by Cinar in [5].

Elsayed [12] has got the solutions of the following systems of the difference equations

$$
x_{n+1}=\frac{x_{n-1}}{ \pm 1+x_{n-1} y_{n}}, y_{n+1}=\frac{y_{n-1}}{\mp 1+y_{n-1} x_{n}} .
$$

Liu et al. [24] obtained the solution of particular cases of the following general system of difference equations

$$
x_{n+1}=\frac{x_{n-1}}{y_{n} x_{n-1}-1}, y_{n+1}=\frac{y_{n-1}}{x_{n} y_{n-1}-1}, z_{n+1}=\frac{1}{y_{n} z_{n-1}} .
$$

Özban [25] has investigated the positive solutions of the system of rational difference equations

$$
x_{n+1}=\frac{a}{y_{n-3}}, y_{n+1}=\frac{b y_{n-3}}{x_{n-q} y_{n-q}} .
$$

In [29] Yalçınkaya investigated the sufficient condition for the global asymptotic stability of the following system of difference equations

$$
z_{n+1}=\frac{t_{n} z_{n-1}+a}{t_{n}+z_{n-1}}, t_{n+1}=\frac{z_{n} t_{n-1}+a}{z_{n}+t_{n-1}} .
$$

Also, Yalçınkaya [30] has obtained the sufficient conditions for the global asymptotic stability of the system of two nonlinear difference equations

$$
x_{n+1}=\frac{x_{n}+y_{n-1}}{x_{n} y_{n-1}-1}, y_{n+1}=\frac{y_{n}+x_{n-1}}{y_{n} x_{n-1}-1} .
$$

Zhang et al.[36] has investigated the positive solutions of the systems

$$
x_{n+1}=A+\frac{y_{n-k}}{y_{n}}, \quad y_{n+1}=A+\frac{x_{n-k}}{x_{n}} .
$$

Similar nonlinear systems of rational difference equations were investigated see [24][34].

Our aim in this paper is to investigate the periodic nature and get the form of the solutions of the following systems of rational difference equations

$$
x_{n+1}=\frac{1 \pm z_{n}}{y_{n-1}}, \quad y_{n+1}=\frac{1 \pm x_{n}}{z_{n-1}}, \quad z_{n+1}=\frac{1 \pm y_{n}}{x_{n-1}} .
$$

with a nonzero real numbers initial conditions.

Definition (Periodicity)

A sequence $\left\{x_{n}\right\}_{n=-k}^{\infty}$ is said to be periodic with period $p$ if $x_{n+p}=x_{n}$ for all $n \geq-k$. 
2 First System: $x_{n+1}=\frac{1+z_{n}}{y_{n-1}}, y_{n+1}=\frac{1+x_{n}}{z_{n-1}}, z_{n+1}=$ $\frac{1+y_{n}}{x_{n-1}}$.

In this part, the solutions of the system of two difference equations have been studied

$$
x_{n+1}=\frac{1+z_{n}}{y_{n-1}}, \quad y_{n+1}=\frac{1+x_{n}}{z_{n-1}}, \quad z_{n+1}=\frac{1+y_{n}}{x_{n-1}}, \quad n=0,1, \ldots,
$$

with nonzero real initials conditions $x_{-1}, x_{0}, y_{-1}, y_{0}, z_{-1}, z_{0}$.

Theorem 1 Suppose that $\left\{x_{n}, y_{n}, z_{n}\right\}$ are solutions of system (1), then the following statements are true:-

1. $x_{n+5}=y_{n}, y_{n+5}=z_{n}, z_{n+5}=x_{n}$ for $n \geq-1$.

2. $x_{n+10}=z_{n}, y_{n+10}=x_{n}, z_{n+10}=y_{n}$ for $n \geq-1$.

3. $\left\{x_{n}\right\}_{n=-1}^{+\infty},\left\{y_{n}\right\}_{n=-1}^{+\infty}$ and $\left\{z_{n}\right\}_{n=-1}^{+\infty}$ are periodic with period ten i.e., $x_{n+15}=$ $x_{n}, y_{n+15}=y_{n}, z_{n+15}=z_{n}$ for $n \geq-1$.

4. We have

$$
\begin{aligned}
x_{10 n-1}=x_{-1}, \quad x_{10 n}=x_{0}, \quad x_{10 n+1}=\frac{1+z_{0}}{y_{-1}}, \quad x_{10 n+2}=\frac{1+y_{0}+x_{-1}}{y_{0} x_{-1}}, \\
x_{10 n+3}=\frac{1+z_{-1}}{x_{0}}, \quad x_{10 n+4}=y_{-1}, \quad x_{10 n+5}=y_{0}, \quad x_{10 n+6}=\frac{1+x_{0}}{z_{-1}}, \\
x_{10 n+7}=\frac{1+z_{0}+y_{-1}}{z_{0} y_{-1}}, \quad x_{10 n+8}=\frac{1+x_{-1}}{y_{0}}, \quad x_{10 n+9}=z_{-1}, \quad x_{10 n+10}=z_{0}, \\
x_{10 n+11}=\frac{1+y_{0}}{x_{-1}}, \quad x_{10 n+12}=\frac{1+x_{0}+z_{-1}}{x_{0} z_{-1}}, \quad x_{10 n+13}=\frac{1+y_{-1}}{z_{0}} .
\end{aligned}
$$

and

$$
\begin{aligned}
y_{10 n-1}=y_{-1}, \quad y_{10 n}=y_{0}, \quad y_{10 n+1}=\frac{1+x_{0}}{z_{-1}}, \quad y_{10 n+2}=\frac{1+z_{0}+y_{-1}}{z_{0} y_{-1}}, \\
y_{10 n+3}=\frac{1+x_{-1}}{y_{0}}, \quad y_{10 n+4}=z_{-1}, \quad y_{10 n+5}=z_{0}, \quad y_{10 n+6}=\frac{1+y_{0}}{x_{-1}}, \\
y_{10 n+7}=\frac{1+x_{0}+z_{-1}}{x_{0} z_{-1}}, \quad y_{10 n+8}=\frac{1+y_{-1}}{z_{0}}, \quad y_{10 n+9}=x_{-1}, \quad y_{10 n+10}=x_{0}, \\
y_{10 n+11}=\frac{1+z_{0}}{y_{-1}}, \quad y_{10 n+12}=\frac{1+y_{0}+x_{-1}}{y_{0} x_{-1}}, \quad y_{10 n+13}=\frac{1+z_{-1}}{x_{0}} .
\end{aligned}
$$

as well 


$$
\begin{aligned}
z_{10 n-1} & =z_{-1}, \quad z_{10 n}=z_{0}, \quad z_{10 n+1}=\frac{1+y_{0}}{x_{-1}}, \quad z_{10 n+2}=\frac{1+x_{0}+z_{-1}}{x_{0} z_{-1}}, \\
z_{10 n+3} & =\frac{1+y_{-1}}{z_{0}}, \quad z_{10 n+4}=x_{-1}, \quad z_{10 n+5}=x_{0}, \quad z_{10 n+6}=\frac{1+z_{0}}{y_{-1}}, \\
z_{10 n+7} & =\frac{1+y_{0}+x_{-1}}{y_{0} x_{-1}}, \quad z_{10 n+8}=\frac{1+z_{-1}}{x_{0}}, \quad z_{10 n+9}=y_{-1}, \quad z_{10 n+10}=y_{0}, \\
z_{10 n+11} & =\frac{1+x_{0}}{z_{-1}}, \quad z_{10 n+12}=\frac{1+z_{0}+y_{-1}}{z_{0} y_{-1}}, \quad z_{10 n+13}=\frac{1+x_{-1}}{y_{0}} .
\end{aligned}
$$

Or equivalently

$$
\begin{aligned}
& \left\{x_{n}\right\}_{n=-1}^{+\infty}=\left\{\begin{array}{c}
\left.x_{-1}, x_{0}, \frac{1+z_{0}}{y_{-1}}, \frac{1+y_{0}+x_{-1}}{y_{0} x_{-1}}, \frac{1+z_{-1}}{x_{0}}, y_{-1}, y_{0}, \frac{1+x_{0}}{z_{-1}}, \frac{1+z_{0}+y_{-1}}{z_{0} y_{-1}},\right\}, \\
\frac{1+x_{-}}{y_{0}}, z_{-1}, z_{0}, \frac{1+y_{0}}{x_{-1}}, \frac{1+x_{0}+z_{-1}}{x_{0} z_{-1}}, \frac{1+y_{-1}}{z_{0}}, x_{-1}, x_{0}, \ldots
\end{array}\right\} \\
& \left\{y_{n}\right\}_{n=-1}^{+\infty}=\left\{\begin{array}{c}
y_{-1}, y_{-1}, \frac{1+x_{0}}{z_{-1}}, \frac{1+z_{0}+y_{-1}}{z_{0} y_{-1}}, \frac{1+x_{-1}}{y_{0}}, z_{-1}, z_{0}, \frac{1+y_{0}}{x_{-1}}, \frac{1+x_{0}+z_{-1}}{x_{0} z_{-1}}, \\
\frac{1+y_{-1}}{z_{0}}, x_{-1}, x_{0} \frac{1+z_{0}}{y_{-1}}, \frac{1+y_{0}+x_{-1}}{y_{0} x_{-1}}, \frac{1+z_{-1}}{x_{0}}, y_{-1}, y_{0}, \ldots
\end{array}\right\}, \\
& \left\{z_{n}\right\}_{n=-1}^{+\infty}=\left\{\begin{array}{c}
z_{-1}, z_{0}, \frac{1+y_{0}}{x_{-1}}, \frac{1+x_{0}+z_{-1}}{x_{0} z_{-1}}, \frac{1+y_{-1}}{z_{0}}, x_{-1}, x_{0}, \frac{1+z_{0}}{y_{-1}}, \frac{1+y_{0}+x_{-1}}{y_{0} x_{-1}}, \\
\frac{1+z_{-1}}{x_{0}}, y_{-1}, y_{0}, \frac{1+x_{0}}{z_{-1}}, \frac{1+z_{0}+y_{-1}}{z_{0} y_{-1}} \frac{1+x_{-1}}{y_{0}}, z_{-1}, z_{0}, \ldots
\end{array}\right\} .
\end{aligned}
$$

Proof: 1. From Eq.(1) we see that

$$
\begin{gathered}
x_{n+5}=\frac{1+z_{n+4}}{y_{n+3}}, \quad y_{n+5}=\frac{1+x_{n+4}}{z_{n+3}}, \quad z_{n+5}=\frac{1+y_{n+4}}{x_{n+3}} \\
x_{n+5}=\frac{1+\left(\frac{1+y_{n+3}}{x_{n+2}}\right)}{\left(\frac{1+x_{n+2}}{z_{n+1}}\right)}, \quad y_{n+5}=\frac{1+\left(\frac{1+z_{n+3}}{y_{n+2}}\right)}{\left(\frac{1+y_{n+2}}{x_{n+1}}\right)}, y_{n+5}=\frac{1+\left(\frac{1+x_{n+3}}{z_{n+2}}\right)}{\left(\frac{1+z_{n+2}}{y_{n+1}}\right)} \\
x_{n+5}=\frac{1+z_{n+1}}{x_{n+2}}, \quad y_{n+5}=\frac{1+x_{n+1}}{y_{n+2}}, \quad z_{n+5}=\frac{1+y_{n+1}}{z_{n+2}}, \\
x_{n+5}=\frac{1+z_{n+1}}{\frac{1+z_{n+1}}{y_{n}}}, y_{n+5}=\frac{1+x_{n+1}}{\frac{1+x_{n+1}}{z_{n}}}, \quad z_{n+5}=\frac{1+y_{n+1}}{\frac{1+y_{n+1}}{x_{n}}} .
\end{gathered}
$$

Therefore

$$
x_{n+5}=y_{n}, \quad y_{n+5}=z_{n}, \quad z_{n+5}=x_{n} .
$$

2. Also, we get

$$
\begin{aligned}
& x_{n+10}=y_{n+5}=z_{n}, \\
& y_{n+10}=z_{n+5}=x_{n},
\end{aligned}
$$




$$
z_{n+10}=x_{n+5}=y_{n}
$$

3. Ditto,

$$
\begin{aligned}
& x_{n+15}=y_{n+10}=z_{n+5}=x_{n}, \\
& y_{n+15}=z_{n+10}=x_{n+5}=y_{n}, \\
& z_{n+10}=x_{n+10}=y_{n+5}=z_{n} .
\end{aligned}
$$

3. For $n=0$ the result holds. Now suppose that $n>0$ and that our assumption holds for $n-1$. That is;

$$
\begin{aligned}
& x_{15 n-16}=x_{-1}, \quad x_{15 n-15}=x_{0}, \quad x_{15 n-14}=\frac{1+z_{0}}{y_{-1}}, \quad x_{15 n-13}=\frac{1+y_{0}+x_{-1}}{y_{0} x_{-1}}, \\
& x_{15 n-12}=\frac{1+z_{-1}}{x_{0}}, \quad x_{15 n-11}=y_{-1}, \quad x_{15 n-10}=y_{0}, \quad x_{15 n-9}=\frac{1+x_{0}}{z_{-1}}, \\
& x_{15 n-8}=\frac{1+z_{0}+y_{-1}}{z_{0} y_{-1}}, \quad x_{15 n-7}=\frac{1+x_{-1}}{y_{0}}, \quad x_{15 n-6}=z_{-1}, \quad x_{15 n-5}=z_{0}, \\
& x_{15 n-4}=\frac{1+y_{0}}{x_{-1}}, \quad x_{15 n-3}=\frac{1+x_{0}+z_{-1}}{x_{0} z_{-1}}, \quad x_{15 n-2}=\frac{1+y_{-1}}{z_{0}} .
\end{aligned}
$$

and

$$
\begin{aligned}
y_{15 n-16} & =y_{-1}, \quad y_{15 n-15}=y_{0}, \quad y_{15 n-14}=\frac{1+x_{0}}{z_{-1}}, \quad y_{15 n-13}=\frac{1+z_{0}+y_{-1}}{z_{0} y_{-1}}, \\
y_{15 n-12} & =\frac{1+x_{-1}}{y_{0}}, \quad y_{15 n-11}=z_{-1}, \quad y_{15 n-10}=z_{0}, \quad y_{15 n-9}=\frac{1+y_{0}}{x_{-1}}, \\
y_{15 n-8} & =\frac{1+x_{0}+z_{-1}}{x_{0} z_{-1}}, \quad y_{15 n-7}=\frac{1+y_{-1}}{z_{0}}, \quad y_{15 n-6}=x_{-1}, \quad y_{15 n-5}=x_{0}, \\
y_{15 n-4} & =\frac{1+z_{0}}{y_{-1}}, \quad y_{15 n-3}=\frac{1+y_{0}+x_{-1}}{y_{0} x_{-1}}, \quad y_{15 n-2}=\frac{1+z_{-1}}{x_{0}} .
\end{aligned}
$$

and the result of $\mathrm{Z}$

$$
\begin{aligned}
z_{15 n-16} & =z_{-1}, \quad z_{15 n-15}=z_{0}, \quad z_{15 n-14}=\frac{1+y_{0}}{x_{-1}}, \quad z_{15 n-13}=\frac{1+x_{0}+z_{-1}}{x_{0} z_{-1}}, \\
z_{15 n-12} & =\frac{1+y_{-1}}{z_{0}}, \quad z_{15 n-11}=x_{-1}, \quad z_{15 n-10}=x_{0}, \quad z_{15 n-9}=\frac{1+z_{0}}{y_{-1}} \\
z_{15 n-8} & =\frac{1+y_{0}+x_{-1}}{y_{0} x_{-1}}, \quad z_{15 n-7}=\frac{1+z_{-1}}{x_{0}}, \quad z_{15 n-6}=y_{-1}, \quad z_{15 n-5}=y_{0}, \\
z_{15 n-4} & =\frac{1+x_{0}}{z_{-1}}, \quad z_{15 n-3}=\frac{1+z_{0}+y_{-1}}{z_{0} y_{-1}}, \quad z_{15 n-2}=\frac{1+x_{-1}}{y_{0}} .
\end{aligned}
$$


Now, it follows from Eq.(1) that

$$
\begin{aligned}
& x_{15 n-1}= \frac{1+z_{15 n-2}}{y_{15 n-3}}=\frac{1+\left(\frac{1+x_{-1}}{y_{0}}\right)}{\left(\frac{1+y_{0}+x_{-1}}{y_{0} x_{-1}}\right)}=\frac{\left(1+y_{0}+x_{-1}\right) x_{-1}}{1+y_{0}+x_{-1}}=x_{-1}, \\
& y_{15 n-1}= \frac{1+x_{15 n-2}}{z_{15 n-3}}=\frac{1+\left(\frac{1+y_{-1}}{z_{0}}\right)}{\left(\frac{1+z_{0}+y_{-1}}{z_{0} y_{-1}}\right)}=\frac{\left(1+z_{0}+y_{-1}\right) y_{-1}}{1+z_{0}+y_{-1}}=y_{-1}, \\
& z_{15 n-1}=\frac{1+y_{15 n-2}}{x_{15 n-3}}=\frac{1+\left(\frac{1+z_{-1}}{x_{0}}\right)}{\left(\frac{1+x_{0}+z_{-1}}{x_{0}}\right)}=\frac{\left(1+x_{0}+z_{-1}\right) z_{-1}}{1+x_{0}+z_{-1}}=z_{-1}, \\
& x_{15 n}=\frac{1+z_{15 n-1}}{y_{15 n-2}}=\frac{1+z_{-1}}{\left(\frac{1+z_{-1}}{x_{0}}\right)}=x_{0}, \\
& y_{15 n}=\frac{1+x_{15 n-1}}{z_{15 n-2}}=\frac{1+x_{-1}}{\left(\frac{1+x_{-1}}{y_{0}}\right)}=y_{0}, \\
& y_{15 n}=\frac{1+y_{15 n-1}}{x_{15 n-2}}=\frac{1+y_{-1}}{\left(\frac{1+y_{-1}}{z_{0}}\right)}=z_{0} . \\
& 1+z_{15 n}=\frac{1+z_{0}}{y_{-1}}, \quad y_{15 n+1}=\frac{1+x_{15 n}}{z_{15 n-1}}=\frac{1+x_{0}}{z_{-1}},, \quad z_{15 n+1}=\frac{1+y_{15 n}}{x_{15 n-1}}=\frac{1+y_{0}}{x_{-1}},
\end{aligned}
$$
also

$$
\begin{gathered}
x_{15 n+2}=\frac{1+z_{15 n+1}}{y_{15 n}}=\frac{1+\left(\frac{1+y_{0}}{x_{-1}}\right)}{y_{0}}=\frac{1+y_{0}+x_{-1}}{y_{0} x_{-1}}, \\
y_{15 n+2}=\frac{1+x_{15 n+1}}{z_{15 n}}=\frac{1+\left(\frac{1+z_{0}}{y_{-1}}\right)}{z_{0}}=\frac{1+z_{0}+y_{-1}}{z_{0} y_{-1}}, \\
z_{15 n+2}=\frac{1+y_{15 n+1}}{x_{15 n}}=\frac{1+\left(\frac{1+x_{0}}{z_{-1}}\right)}{x_{0}}=\frac{1+x_{0}+z_{-1}}{x_{0} z_{-1}} \\
x_{15 n+3}=\frac{1+z_{15 n+2}}{y_{15 n+1}}=\frac{1+\left(\frac{1+x_{0}+z_{-1}}{x_{0} z_{-1}}\right)}{\frac{1+x_{0}}{z_{-1}}}=\frac{x_{0}\left(z_{-1}+1\right)+\left(1+z_{-1}\right)}{x_{0}\left(1+x_{0}\right)}=\frac{1+z_{-1}}{x_{0}} \\
y_{15 n+3}=\frac{1+x_{15 n+2}}{z_{15 n+1}}=\frac{1+\left(\frac{1+y_{0}+x_{-1}}{y_{0} x_{-1}}\right)}{\frac{1+y_{0}}{x_{-1}}}=\frac{y_{0}\left(x_{-1}+1\right)+\left(1+x_{-1}\right)}{y_{0}\left(1+y_{0}\right)}=\frac{1+x_{-1}}{y_{0}}, \\
z_{15 n+3}=\frac{1+y_{15 n+2}}{x_{15 n+1}}=\frac{1+\left(\frac{1+z_{0}+y_{-1}}{z_{0} y_{-1}}\right)}{\frac{1+z_{0}}{y_{-1}}}=\frac{z_{0}\left(y_{-1}+1\right)+\left(1+y_{-1}\right)}{z_{0}\left(1+z_{0}\right)}=\frac{1+y_{-1}}{z_{0}} .
\end{gathered}
$$


Identically, other relations may easily be proven. The proof is done.

The following theorem can be proves similarly.

3 Second System: $x_{n+1}=\frac{1-z_{n}}{y_{n-1}}, y_{n+1}=\frac{1-x_{n}}{z_{n-1}}, z_{n+1}=$ $\frac{1-y_{n}}{x_{n-1}}$

In this part, the solutions of the system of two difference equations have been examined

$$
x_{n+1}=\frac{1-z_{n}}{y_{n-1}}, \quad y_{n+1}=\frac{1-x_{n}}{z_{n-1}}, \quad z_{n+1}=\frac{1-y_{n}}{x_{n-1}}, \quad n=0,1, \ldots,
$$

with a nonzero real numbers initial conditions.

Theorem 2 Suppose that $\left\{x_{n}, y_{n}, z_{n}\right\}$ are solutions of system (2). Then

1. $x_{n+5}=y_{n}, y_{n+5}=z_{n}, z_{n+5}=x_{n}$ for $n \geq-1$.

2. $x_{n+10}=z_{n}, y_{n+10}=x_{n}, z_{n+10}=y_{n}$ for $n \geq-1$.

3. $\left\{x_{n}\right\}_{n=-1}^{+\infty}$ and $\left\{y_{n}\right\}_{n=-1}^{+\infty}$ and $\left\{z_{n}\right\}_{n=-1}^{+\infty}$ are periodic with period ten. i.e., $x_{n+15}=$ $x_{n}, y_{n+15}=y_{n}, z_{n+15}=z_{n}$ for $n \geq-1$ and the solutions takes the form

$$
\begin{aligned}
x_{10 n-1}=x_{-1}, \quad x_{10 n}=x_{0}, \quad x_{10 n+1}=\frac{1-z_{0}}{y_{-1}}, \quad x_{10 n+2}=\frac{-1+y_{0}+x_{-1}}{y_{0} x_{-1}}, \\
x_{10 n+3}=\frac{1-z_{-1}}{x_{0}}, \quad x_{10 n+4}=y_{-1}, \quad x_{10 n+5}=y_{0}, \quad x_{10 n+6}=\frac{1-x_{0}}{z_{-1}}, \\
x_{10 n+7}=\frac{-1+z_{0}+y_{-1}}{z_{0} y_{-1}}, \quad x_{10 n+8}=\frac{1-x_{-1}}{y_{0}}, \quad x_{10 n+9}=z_{-1}, \quad x_{10 n+10}=z_{0}, \\
x_{10 n+11}=\frac{1-y_{0}}{x_{-1}}, \quad x_{10 n+12}=\frac{-1+x_{0}+z_{-1}}{x_{0} z_{-1}}, \quad x_{10 n+13}=\frac{1-y_{-1}}{z_{0}} . \\
y_{10 n-1}=y_{-1}, \quad y_{10 n}=y_{0}, \quad y_{10 n+1}=\frac{1-x_{0}}{z_{-1}}, \quad y_{10 n+2}=\frac{-1+z_{0}+y_{-1}}{z_{0} y_{-1}}, \\
y_{10 n+3}=\frac{1-x_{-1}}{y_{0}}, \quad y_{10 n+4}=z_{-1}, \quad y_{10 n+5}=z_{0}, \quad y_{10 n+6}=\frac{1-y_{0}}{x_{-1}}, \\
y_{10 n+7}=\frac{-1+x_{0}+z_{-1}}{x_{0} z_{-1}}, \quad y_{10 n+8}=\frac{1-y_{-1}}{z_{0}}, \quad y_{10 n+9}=x_{-1}, \quad y_{10 n+10}=x_{0}, \\
y_{10 n+11}=\frac{1-z_{0}}{y_{-1}}, \quad y_{10 n+12}=\frac{-1+y_{0}+x_{-1}}{y_{0} x_{-1}}, \quad y_{10 n+13}=\frac{1-z_{-1}}{x_{0}} .
\end{aligned}
$$

and 


$$
\begin{aligned}
z_{10 n-1} & =z_{-1}, \quad z_{10 n}=z_{0}, \quad z_{10 n+1}=\frac{1-y_{0}}{x_{-1}}, \quad z_{10 n+2}=\frac{-1+x_{0}+z_{-1}}{x_{0} z_{-1}}, \\
z_{10 n+3} & =\frac{1-y_{-1}}{z_{0}}, \quad z_{10 n+4}=x_{-1}, \quad z_{10 n+5}=x_{0}, \quad z_{10 n+6}=\frac{1-z_{0}}{y_{-1}} \\
z_{10 n+7} & =\frac{-1+y_{0}+x_{-1}}{y_{0} x_{-1}}, \quad z_{10 n+8}=\frac{1-z_{-1}}{x_{0}}, \quad z_{10 n+9}=y_{-1}, \quad z_{10 n+10}=y_{0}, \\
z_{10 n+11} & =\frac{1-x_{0}}{z_{-1}}, \quad z_{10 n+12}=\frac{-1+z_{0}+y_{-1}}{z_{0} y_{-1}}, \quad z_{10 n+13}=\frac{1-x_{-1}}{y_{0}} .
\end{aligned}
$$

Or equivalently

$$
\begin{aligned}
& \left\{x_{n}\right\}_{n=-1}^{+\infty}=\left\{\begin{array}{c}
x_{-1}, x_{0}, \frac{1-z_{0}}{y_{-1}}, \frac{-1+y_{0}+x_{-1}}{y_{0} x_{-1}}, \frac{1-z_{-1}}{x_{0}}, y_{-1}, y_{0}, \frac{1-x_{0}}{z_{-1}}, \frac{-1+z_{0}+y_{-1}}{z_{0} y_{-1}}, x_{-1}, z_{-1}, z_{0}, \frac{1-y_{0}}{x_{-}}, \frac{-1+x_{0}+z_{-1}}{x_{0} z_{-1}}, \frac{1-y_{-1}}{z_{0}}, x_{-1}, x_{0}, \ldots
\end{array},\right. \\
& \left\{y_{n}\right\}_{n=-1}^{+\infty}=\left\{\begin{array}{c}
y_{-1}, y_{-1}, \frac{1-x_{0}}{z_{-}}, \frac{-1+z_{0}+y_{-1}}{z_{0} y_{-1}}, \frac{1-x_{-1}}{y_{0}}, z_{-1}, z_{0}, \frac{1-y_{0}}{x_{-1}}, \frac{-1+x_{0}+z_{-1}}{x_{0} z_{-1}}, \\
\frac{1-y_{-1}}{z_{0}}, x_{-1}, x_{0} \frac{1-z_{0}}{y_{-1}}, \frac{-1+y_{0}+x_{-1}}{y_{0} x_{-1}}, \frac{1-z_{-1}}{x_{0}}, y_{-1}, y_{0}, \ldots
\end{array}\right\}, \\
& \left\{z_{n}\right\}_{n=-1}^{+\infty}=\left\{\begin{array}{c}
z_{-1}, z_{0}, \frac{1-y_{0}}{x_{-1}}, \frac{-1+x_{0}+z_{-1}}{x_{0} z_{-1}}, \frac{1-y_{-1}}{z_{0}}, x_{-1}, x_{0}, \frac{1-z_{0}}{y_{-1}}, \frac{-1+y_{0}+x_{-1}}{y_{0} x_{-1}}, \\
\frac{1-z_{-1}}{x_{0}}, y_{-1}, y_{0}, \frac{1-x_{0}}{z_{-1}}, \frac{-1+z_{0}+y_{-1}}{z_{0} y_{-1}} \frac{1-x_{-1}}{y_{0}}, z_{-1}, z_{0}, \ldots
\end{array}\right\} .
\end{aligned}
$$

\section{$4 \quad$ Numerical Examples}

Significant numerical examples have been considered in this part in order to show the results obtained earlier, and also to enhance our theoretical discussion.

Example 1. Consider the difference system equation (1) with the initial conditions $x_{-1}=0.2, x_{0}=0.6, y_{-1}=3, y_{0}=7, z_{-1}=-5$ and $z_{0}=-1.6$. (See Fig. 1 ). 


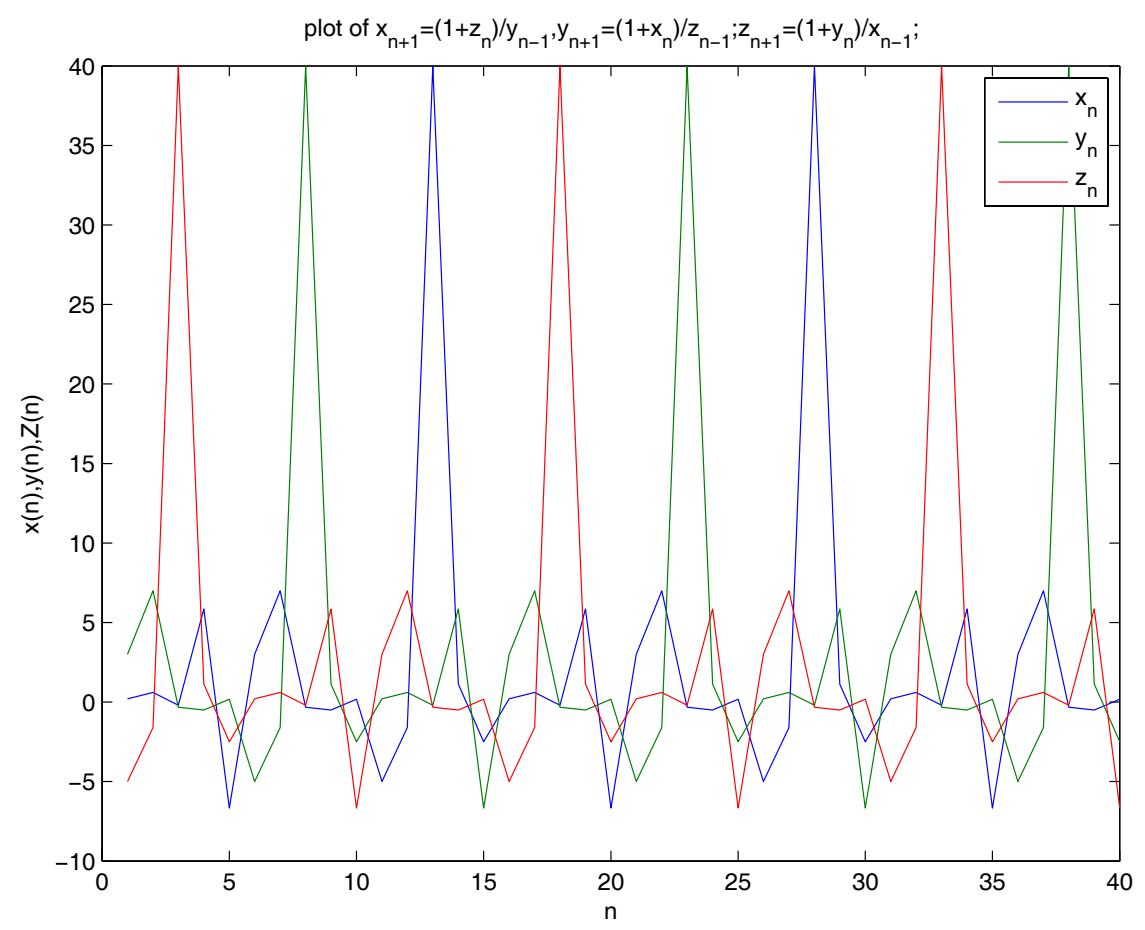

Figure 1. This figure shows the solutions of the system

$$
x_{n+1}=\frac{1+z_{n}}{y_{n-1}}, \quad y_{n+1}=\frac{1+x_{n}}{z_{n-1}}, \quad z_{n+1}=\frac{1+y_{n}}{x_{n-1}},
$$

where $x_{-1}=0.2, x_{0}=0.6, y_{-1}=3, y_{0}=7, z_{-1}=-5$ and $z_{0}=-1.6$.

Example 2. For the initial conditions $x_{-1}=9, x_{0}=-0.2, y_{-1}=2, y_{0}=0.7$, $z_{-1}=6$ and $z_{0}=1.6$. when we take the system (1). (See Fig. 2). 


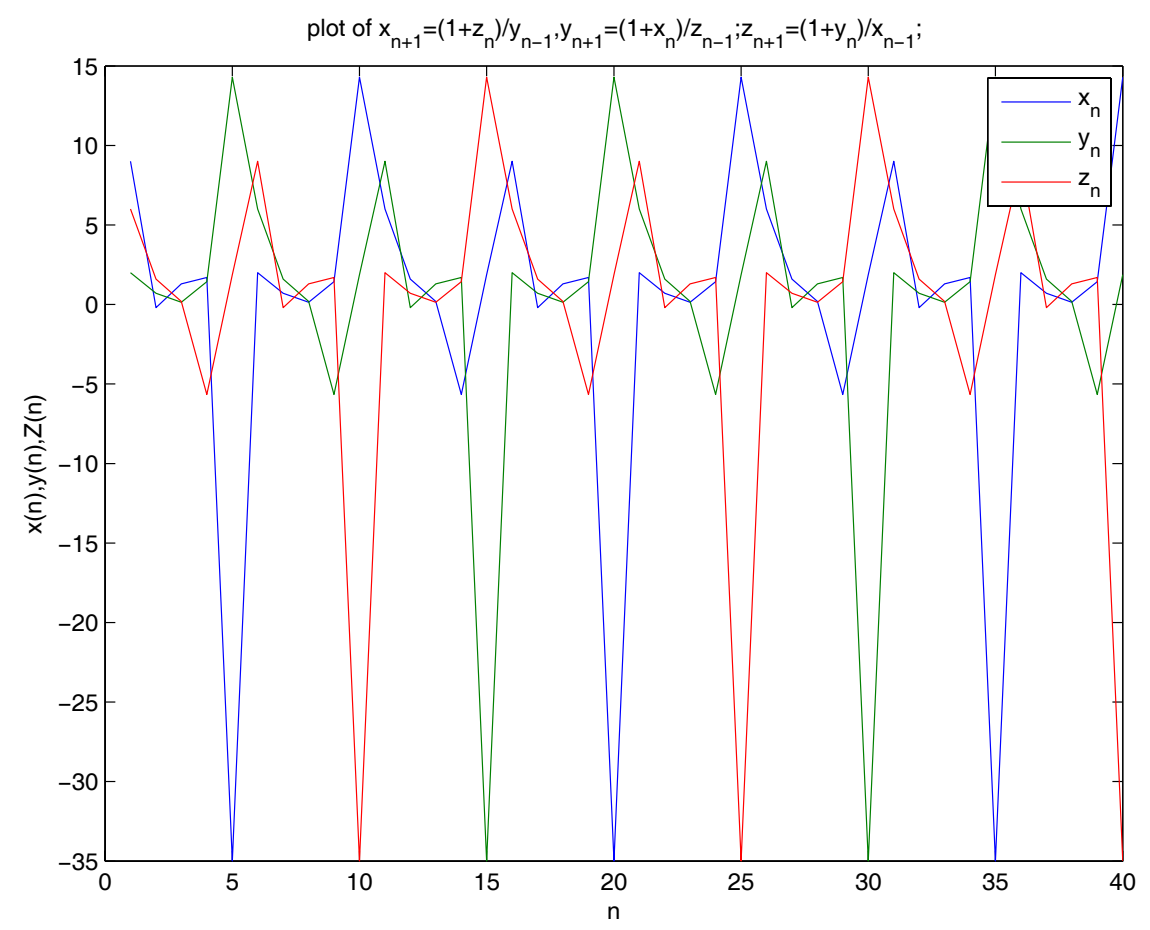

Figure 2. This figure shows the periodicity of system (1)

where $x_{-1}=9, x_{0}=-0.2, y_{-1}=2, y_{0}=0.7, z_{-1}=6$ and $z_{0}=1.6$.

Example 3. Take the initial conditions as follows $x_{-1}=9, x_{0}=-0.2, y_{-1}=2$, $y_{0}=0.7, z_{-1}=6$ and $z_{0}=1.6$ for the difference system equation (2). See Fig. 3. 


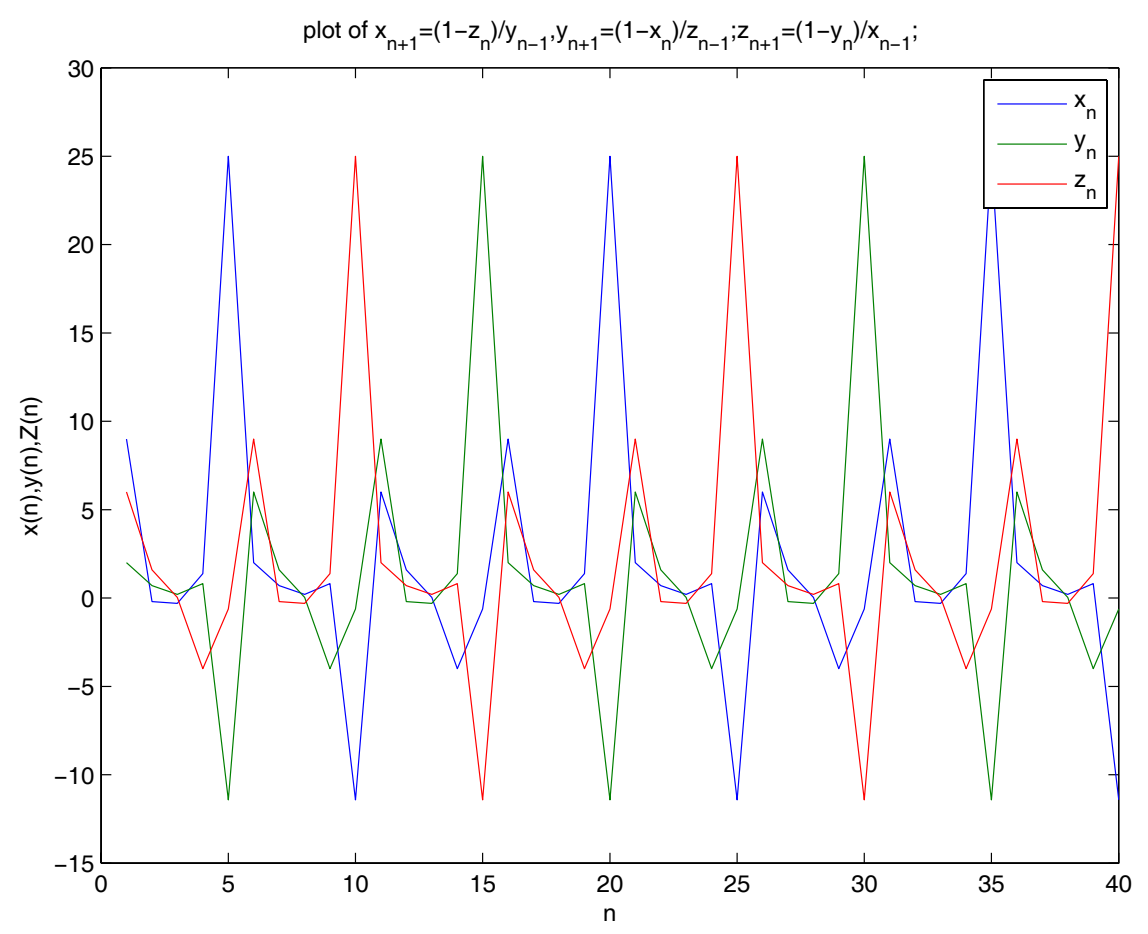

Figure 3. This figure shows the periodicity of the solutions of the system

$$
\begin{gathered}
x_{n+1}=\frac{1-z_{n}}{y_{n-1}}, y_{n+1}=\frac{1-x_{n}}{z_{n-1}}, z_{n+1}=\frac{1-y_{n}}{x_{n-1}} \\
\text { where } x_{-1}=9, x_{0}=-0.2, y_{-1}=2, y_{0}=0.7, z_{-1}=6 \text { and } z_{0}=1.6 .
\end{gathered}
$$

Example 4. Put the initial conditions $x_{-1}=9, x_{0}=2, y_{-1}=0.2, y_{0}=-3$, $z_{-1}=-1.4$ and $z_{0}=4$ in the system (2). (See Fig. 4). 


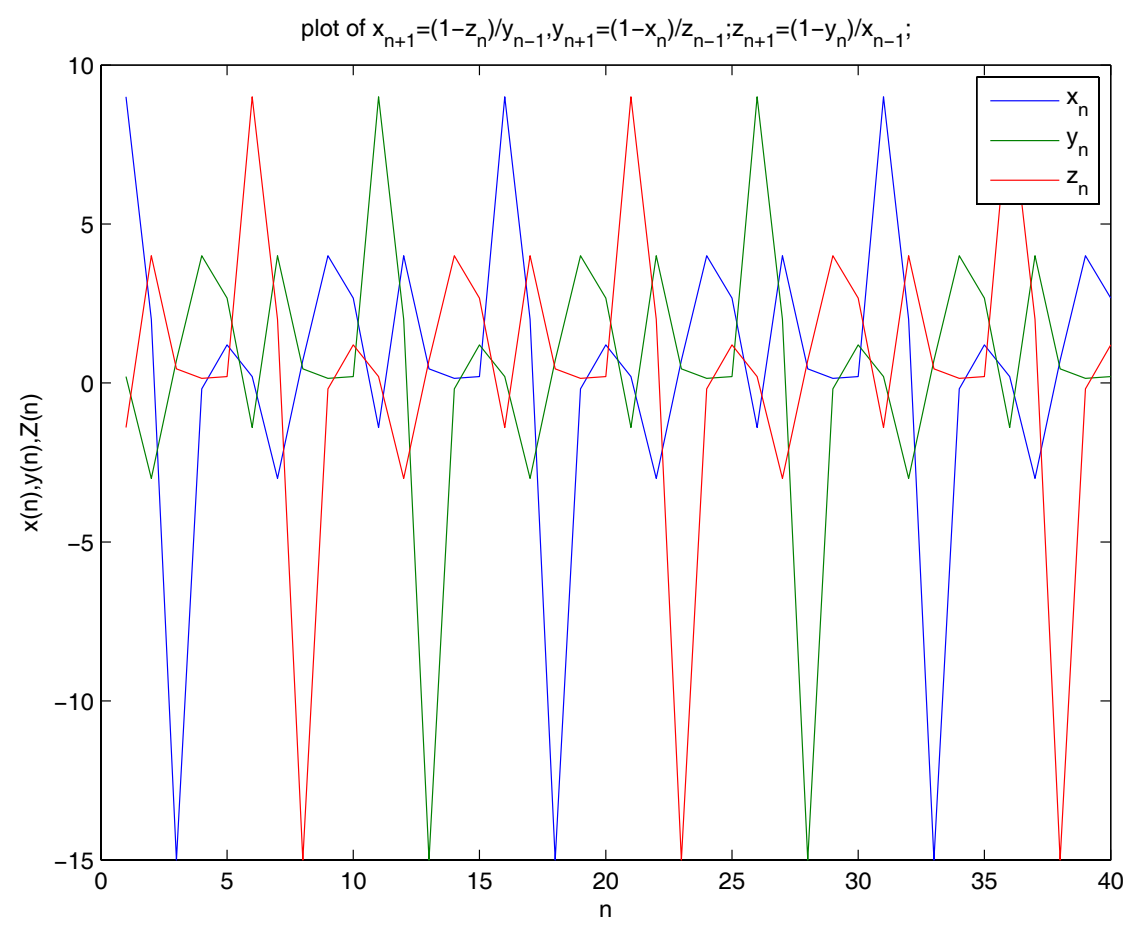

Figure 4. This figure shows the solutions of the system (2), where $x_{-1}=9, x_{0}=2, y_{-1}=0.2, y_{0}=-3, z_{-1}=-1.4$ and $z_{0}=4$.

\section{References}

[1] A. M. Ahmed, On the dynamics of a system of rational difference equation, Dynamics of Continuous, Discrete and Impulsive Systems Series A Mathematical Analysis, 21 (2014), 487-506.

[2] M. Alghamdi, E. M. Elsayed and M. M. El-Dessoky, On the Solutions of Some Systems of Second Order Rational Difference Equations, Life Sci J., 10 (3) (2013), 344-351.

[3] S. Araci, Existence and multiplicity of positive solutions for boundary-value problems of non-linear fractional differential equations, Proc. Jangjeon Math. Soc., 17 (2) (2014), 247-258.

[4] A. Asiri, E. M. Elsayed, and M. M. El-Dessoky, On the Solutions and Periodic Nature of Some Systems of Difference Equations, Journal of Computational and Theoretical Nanoscience, 12 (2015), 1-8.

[5] H. Bao, On a System of Second-Order Nonlinear difference Equations, Journal of Applied Mathematics and Physics, 3 (2015), 903-910. 
[6] C. Cinar, I. Yalçinkaya and R. Karatas, On the positive solutions of the difference equation system $x_{n+1}=m / y_{n}, y_{n+1}=p y_{n} / x_{n-1} y_{n-1}$, J. Inst. Math. Comp. Sci., 18 (2005), 135-136.

[7] Q. Din, On a system of rational difference equation, Demonstratio Mathematica Vol. XLVII (2) (2014), 324-335.

[8] Q. Din, and E. M. Elsayed, Stability analysis of a discrete ecological model, Computational Ecology and Software 4 (2) (2014), 89-103.

[9] E. M. Elabbasy and E. M. Elsayed, Global attractivity and periodic nature of a difference equation, World Appl. Sci. J., 12 (1) (2011), 39-47.

[10] M. M. El-Dessoky, and E. M. Elsayed, On the solutions and periodic nature of some systems of rational difference equations, J. Comput. Anal. Appl., 18 (2) (2015), 206-218.

[11] E. M. Elsayed, On the solutions of a rational system of difference equations, Fasciculi Mathematici, 45 (2010), 25-36.

[12] E. M. Elsayed, Solutions of rational difference system of order two, Math. Comput. Mod., 55 (2012), 378-384.

[13] E. M. Elsayed, Behavior and expression of the solutions of some rational difference equations, J. Comput. Anal. Appl., 15 (1) (2013), 73-81.

[14] E. M. Elsayed, Solution for systems of difference equations of rational form of order two, Comput. Appl. Math., 33 (3) (2014), 751-765.

[15] E. M. Elsayed, On the solutions and periodic nature of some systems of difference equations, Int. J. Biomath., 7 (6) (2014), 1450067, (26 pages).

[16] E. M. Elsayed, New method to obtain periodic solutions of period two and three of a rational difference equation, Nonlinear Dynamics 79 (1) (2015), 241-250.

[17] E. M. Elsayed and H. El-Metwally, Stability and solutions for rational recursive sequence of order three, J. Comput. Anal. Appl., 17 (2) (2014), 305-315.

[18] E. M. Elsayed and H. El-Metwally, Global behavior and periodicity of some difference equations, J. Comput. Anal. Appl., 19 (2) (2015), 298-309.

[19] H. El-Metwally and E. M. Elsayed, Form of solutions and periodicity for systems of difference equations, J. Comput. Anal. Appl., 15(5) (2013), 852-857.

[20] E. A. Grove and G. Ladas, Periodicities in Nonlinear Difference Equations, Chapman \& Hall / CRC Press, 2005.

[21] T. F. Ibrahim and N. Touafek, On a third order rational difference equation with variable coefficients, Dyn. Cont. Disc. Impu. Syst., Appl. Algo., 20 (2013) 251-264. [22] A. S. Kurbanli, C. Cinar and I. Yalçınkaya, On the behavior of positive solutions of the system of rational difference equations, Math. Comp. Mod., 53 (2011), 12611267.

[23] A. Kurbanli, C. Cinar and M. Erdoğan, On the behavior of solutions of the system of rational difference equations $x_{n+1}=\frac{x_{n-1}}{x_{n-1} y_{n}-1}, y_{n+1}=\frac{y_{n-1}}{y_{n-1} x_{n}-1}, \quad z_{n+1}=\frac{x_{n}}{z_{n-1} y_{n}}$, Applied Mathematics 2 (2011), 1031-1038.

[24] K. Liu, P. Li and W. Zhong, On a system of rational difference equations $x_{n+1}=$ $x_{n-1} / y_{n} x_{n-1}-1, y_{n+1}=y_{n-1} / x_{n} y_{n-1}-1, z_{n+1}=1 / y_{n} z_{n-1}$ Fasciculi Mathematici, 51 
(2013), 105-114.

[25] A. Y. Ozban, On the system of rational difference equations $x_{n+1}=a / y_{n-3}$, $y_{n+1}=b y_{n-3} / x_{n-q} y_{n-q}$, Appl. Math. Comp., 188(1) (2007), 833-837.

[26] M. N. Qureshi, A. Q. Khan and Q. Din, Global behavior of third order system of rational difference equations, Int. J. Eng. Res. Tech., 2 (5) (2013), 2182-2191.

[27] M. Mansour, M. M. El-Dessoky and E. M. Elsayed, On the solution of rational systems of difference equations, J. Comput. Anal. Appl., 15 (5) (2013), 967-976.

[28] N. Touafek and E. M. Elsayed, On a second order rational systems of difference equation, Hokkaido Mathematical Journal, 44 (1) (2015), 29-45..

[29] I. Yalçınkaya, On the global asymptotic stability of a second-order system of difference equations, Disc. Dyn. Nat. Soc., Vol. 2008, Article ID 860152, 12 pages.

[30] I. Yalçınkaya, On the global asymptotic behavior of a system of two nonlinear difference equations, ARS Combinatoria, 95 (2010), 151-159.

[31] I. Yalçınkaya, C. Cinar and M. Atalay, On the solutions of systems of difference equations, Advances in Difference Equations, Vol. 2008, Article ID 143943, 9 pages, doi: $10.1155 / 2008 / 143943$.

[32] X. Yang, On the system of rational difference equations $x_{n}=A+y_{n-1} / x_{n-p} y_{n-q}, y_{n}=$ $A+x_{n-1} / x_{n-r} y_{n-s}$, J. Math. Anal. Appl., 307 (2005), 305-311.

[33] X. Yang, Y. Liu and S. Bai, On the system of high order rational difference equations $x_{n}=a / y_{n-p}, y_{n}=b y_{n-p} / x_{n-q} y_{n-q}$, Appl. Math. Comp., 171(2) (2005), 853-856.

[34] E. M. E. Zayed and M. A. El-Moneam, On the rational recursive sequence $x_{n+1}=$ $a x_{n}-\frac{b x_{n}}{c x_{n}-d x_{n-k}}$, Comm. Appl. Nonlinear Analysis, 15 (2008), 47-57.

[35] D. Zhang, W. Ji, L. Wang, and X. Li, On the Symmetrical System of Rational Difference Equation $x_{n+1}=A+y_{n-k} / y_{n}, y_{n+1}=A+x_{n-k} / x_{n}$, Applied Mathematics, 4 (2013), 834-837.

[36] O.Zkan and A.S.Kurbanli, On a system of difference equation, Dis. Dyn. Net. Soc., Volume 2013 (2013), Article ID 970316, 7 pages. 